\title{
SGIENTOMETRIC STUDY OF SCIENTIFIC LITERATURE ON THE ESTUARIES OF CEARÁ STATE
}

\author{
Estudo cienciométrico da produção científica sobre estuários \\ do Estado do Ceará
}

\author{
Samuel Soares Valentim¹, Maria Ozilea Bezerra Menezes²
}

\begin{abstract}
Estuaries are one of most important coastal environmental system it has been a subject of study worldwide. Many researches have been done on estuarine dynamics, especially regarding the circulation and mixing process, in order to understand the principles of estuarine physical oceanography. However the knowledge on estuaries of Ceará state about hydrodynamics conditions has a significant unequal distribution of studies realized over different sectors of the Ceará coast. An inventory was realized to select the main publications available from estuaries of Ceara state inside physical oceanography area. Resulting in a total of 100 publications. These publications were classified as Grey and White literature and the type of literature is associated with low or high repercussion in the academic community. Results showed that $77 \%$ was classified as Grey literature and 23\% White literature. 52\% of studies were realized on estuaries located in east coast of Ceara state (sector 3), mainly in the Jaguaribe river by the great importance of this system, 22\% in the west and extreme west coast (sector 1), 21\% in the metropolitan region of Fortaleza (sector 2) and 5\% are studies in more than one estuaries or sector of coast. There was a historical evolution of research in the estuaries of Ceara, with increase in the number of published studies and consequently a higher level of knowledge, mainly in the Jaguaribe River basin.
\end{abstract}

Keywords: state of knowledge, literature impact, critical review, estuary.

\section{RESUMO}

Os estuários são ambientes costeiros de grande importância econômica, social e ambiental ao redor do Mundo. Muitos estudos têm sido realizados sobre dinâmica estuarina, principalmente relacionado com circulação e processo de mistura para melhor compreensão dos princípios da oceanografia física estuarina. A distribuição dos estudos realizados nos estuários do Estado do Ceará relacionado com a área de hidrodinâmica estuarina apresenta uma desigualdade e/ou um maior interesse pelo principal sistema estuarino da região, o rio Jaguaribe, o que reflete em quantidades distintas de estudos entre os três setores da costa cearense. Ao todo foram selecionados 100 estudos publicados para realizar uma distribuição dos estudos em relação o setor da costa e classificação em Literatura Cinza e Literatura Branca. Os resultados mostram que $77 \%$ das publicações são classificadas como Literatura Cinza e 23\% como Literatura Branca. 52\% dos estudos foram realizados em estuários localizados no litoral leste (setor 3), sendo 42 estudos no estuário do rio Jaguaribe, $22 \%$ nos estuários do litoral oeste e extremo oeste (setor 1), 21\% nos estuários da região metropolitana de Fortaleza (setor 2), e, 5\% são estudos realizados em mais de um estuário e/ou setor do litoral.

Palavras-chaves: estado do conhecimento, Literatura de impacto, revisão crítica, estuário.

\footnotetext{
${ }^{1}$ Aluno de Doutorado em Ciências Marinhas Tropicais, Programa de Pós Graduação em Ciências Marinhas Tropicais. Instituto de Ciências Marinhas, Universidade Federal do Ceará. Av. da Abolição, 3207. Meireles. Fortaleza, CE 60165-08. E-mail: samuelvalen@ yahoo.com.br.

2 Professora Adjunta da Universidade Federal do Ceará, Instituto de Ciências do Mar, Av. da Abolição, 3207. Meireles. Fortaleza, CE 60165-08. E-mail: ozilea@gmail.com
} 


\section{INTRODUCTION}

Scientometry, known as quality research of scientific production, began in the 1960's within UNESCO and Organization for Cooperation and Economic development (OCED), who created methods to evaluate scientific and technological activities (Spinak, 1998). Estuaries are considered important bodies of water not only for environmental services, but also for their value in economy (ports, fishery, and aquaculture) and social aspects (community development). For this reason, to understand estuaries' circulation and mixture processes is a fundamental step to apply the precautionary principle, in other words, to provide the necessary knowledge to analyze scenarios of environmental impacts such as transport of pollutants, hypersalinization conditions, decline of number's species, water quality.

In the last 60 years, many definitions and classifications of estuaries were developed to cover the characteristics of these environments throughout the world (Pritchard, 1952; 1955; 1956; 1960; 1967; Fairbrigde, 1980; Kjerfve, 1986; Dyer, 1991, 1997). The majority of the definitions of estuaries were based on the conditions of estuaries in temperate regions of the northern hemisphere however such definitions negligence features as hypersaline conditions during dry periods or estuaries that periodic closure of their mouths (Day, 1980). Potter et al. (2010) created definition with main characteristic of all estuaries: "An estuary is a partially enclosed coastal body of water that is either permanently or periodically open to the sea and which receives at least periodic discharge from a river(s), and thus, while its salinity is typically less than that of natural sea water and varies temporally and along its length, it can become hypersaline in regions when evaporative water loss is high and freshwater and tidal inputs are negligible"

The Ceará State has a 573-km long coastline and is divided into three sectors: I - extreme west and western coast; 2 - metropolitan region of Fortaleza; 3 - eastern coast. Its estuaries are under semiarid climate conditions which are the predominant feature in 95 per cent of the state's watershed (Campos et al. 2000). In addition, all of them have been significantly impacted by dams built in medium and high river courses (Morais \& Pinheiro, 2011). The main characteristics of the rivers on Northeastern region, where Ceará State is located, are intermittent rivers, wet and dry season (no rain) and Dam built to maintain sufficient water resources for consumption and human activities.

Studies realized on estuaries of Ceará state based on hydrodynamics conditions have difference distribution quantification over east coast and other sectors of Ceará coast. An inventory selected one-hundred (100) publications about estuarine dynamic (Table 1). The goal of this paper is to quantify the studies on estuaries in Ceará state with focus in physical oceanography and then to classify these research per type of literature (grey or white literature).

Table 1 - Inventory of studies realized on estuaries of Ceara State, Northeastern, Brazil.

\begin{tabular}{|c|c|c|c|c|c|c|}
\hline Year & Author(s) & Title & Estuaries & Location & Means of publication & Classification \\
\hline 1974 & $\begin{array}{l}\text { MORAIS, J.O \& } \\
\text { PITOMBEIRA, E. S. }\end{array}$ & $\begin{array}{l}\text { Processos migratórios da } \\
\text { embocadura do rio Maceiozinho } \\
\text { (For-CE-Brasil) }\end{array}$ & $\begin{array}{l}\text { Maceio- } \\
\text { zinho }\end{array}$ & Sector 2 & $\begin{array}{l}\text { Boletim Ciências do Mar/ } \\
\text { UFC }\end{array}$ & $\begin{array}{l}\text { White } \\
\text { literature }\end{array}$ \\
\hline 1976 & PITOMBEIRA, E. S. & $\begin{array}{l}\text { Comporamento migratório a } \\
\text { embocadura e estuário do rio } \\
\text { Coreaú (CE-Brasil) }\end{array}$ & Coreaú & Sector 1 & $\begin{array}{l}\text { Arquivos Ciências do } \\
\text { Mar / UFC }\end{array}$ & $\begin{array}{l}\text { White } \\
\text { literature }\end{array}$ \\
\hline 1985 & $\begin{array}{l}\text { VASCONCELOS, } \\
\text { F. P. \& FREIRE, G. } \\
\text { F. S. }\end{array}$ & $\begin{array}{l}\text { Estudo preliminar dos aspectos } \\
\text { hidrodinâmicos e } \\
\text { sedimentológicos do estuário do } \\
\text { rio Cocó, CE. }\end{array}$ & Cocó & Sector 2 & $\begin{array}{c}\text { Arquivos Ciências do } \\
\text { Mar / UFC }\end{array}$ & $\begin{array}{l}\text { White } \\
\text { literature }\end{array}$ \\
\hline 1988 & MORAIS, J. O. & $\begin{array}{l}\text { Hidrodinâmica e sedimentação no } \\
\text { estuário do rio Pacoti, CE }\end{array}$ & Pacoti & Sector 2 & $\begin{array}{l}\text { Simpósio Brasileiro para } \\
\text { o progresso da Ciência / } \\
\text { SBPC }\end{array}$ & Grey literature \\
\hline 1989 & FREIRE, G; S. S. & $\begin{array}{c}\text { Etude hydrologique e } \\
\text { sedimentologigue de l'estuarire du } \\
\text { Rio Pacoti }\end{array}$ & Pacoti & Sector 2 & These de Doctorat & Grey literature \\
\hline 1991 & $\begin{array}{l}\text { FREIRE, G; S. S. \& } \\
\text { MAIA, L. P. }\end{array}$ & $\begin{array}{l}\text { Regime hidrodinâmico do estuário } \\
\text { do rio Pacoti }\end{array}$ & Pacoti & Sector 2 & Revista de Geologia & $\begin{array}{l}\text { White } \\
\text { literature }\end{array}$ \\
\hline 1991 & $\begin{array}{l}\text { FREIRE, G; S. S., } \\
\text { MAIA, L. P. \& } \\
\text { CASTELO } \\
\text { BRANCO, M. P. N. }\end{array}$ & $\begin{array}{l}\text { Frente salina e balanço do sal no } \\
\text { estuário do rio Pacoti-CE }\end{array}$ & Pacoti & Sector 2 & Revista de Geologia & $\begin{array}{l}\text { White } \\
\text { literature }\end{array}$ \\
\hline
\end{tabular}


(continuação Tabela 1)

\begin{tabular}{|c|c|c|c|c|c|c|}
\hline 1992 & $\begin{array}{l}\text { FREIRE, G; S. S., } \\
\text { MAIA, L. P. \& } \\
\text { MORAIS, J. O. }\end{array}$ & $\begin{array}{c}\text { Natureza do material em } \\
\text { suspensão do estuário do rio } \\
\text { Pacoti }\end{array}$ & Pacoti & Sector 2 & Revista de Geologia & $\begin{array}{l}\text { White } \\
\text { literature }\end{array}$ \\
\hline 1996 & MORAIS, J. O. & $\begin{array}{l}\text { Estudo correntometrico preliminar } \\
\text { do rio Ceará }\end{array}$ & Ceará & Sector 2 & $\begin{array}{c}\text { I Semana Universitária / } \\
\text { UECE }\end{array}$ & Grey literature \\
\hline 1999 & $\begin{array}{l}\text { MORAIS, J. O. \& } \\
\text { PINHEIRO, L. S. }\end{array}$ & $\begin{array}{l}\text { The impacts of the Jaguaribe river } \\
\text { flow on the erosion and } \\
\text { sedimentation of Pontal de Macéio } \\
\text { - State of Ceará - NE Brazil }\end{array}$ & Jaguaribe & Sector 3 & VII ABEQUA & Grey literature \\
\hline 1999 & $\begin{array}{l}\text { MORAIS, J. O. \& } \\
\text { CAVALCANTE, A. } \\
\text { A. }\end{array}$ & $\begin{array}{l}\text { Diagnóstico hidrossedimentológico } \\
\text { fluvial e impactos ambientais no } \\
\text { baixo curso do rio Jaguaribe-CE }\end{array}$ & Jaguaribe & Sector 3 & $\begin{array}{c}\text { V Encontro } \\
\text { Pesquisadores /UECE }\end{array}$ & Grey literature \\
\hline 2000 & $\begin{array}{l}\text { CAVALCANTE et } \\
\text { al. }\end{array}$ & $\begin{array}{c}\text { Interação de fluxos fluviais, } \\
\text { estuarinos e costeiros na } \\
\text { compartimentação da foz do rio } \\
\text { Jaguaribe-CE }\end{array}$ & Jaguaribe & Sector 3 & $\begin{array}{l}\text { XVIII Simpósio de } \\
\text { Geologia do Nordeste }\end{array}$ & Grey literature \\
\hline 2000 & $\begin{array}{l}\text { MORAIS, J. O. \& } \\
\text { CAVALCANTE, A. } \\
\text { A }\end{array}$ & $\begin{array}{l}\text { Estudo preliminar da } \\
\text { caracterização } \\
\text { hidrossedimentológica associada } \\
\text { aos componentes ambientais do } \\
\text { baixo curso do Rio Jaguaribe-CE }\end{array}$ & Jaguaribe & Sector 3 & $52^{\circ}$ Reunião SBPC/UNB * & Grey lite \\
\hline 2000 & $\begin{array}{l}\text { CAVALCANTE, } \\
\text { A. A \& MORAIS, J. } \\
\text { O.. }\end{array}$ & $\begin{array}{l}\text { Descarga sólida no baixo curso do } \\
\text { rio Jaguaribe-CE: Uma abordagem } \\
\text { hidrossedimentológica no contexto } \\
\text { geoambiental }\end{array}$ & Jaguaribe & Sector 3 & $\begin{array}{l}\text { IV Encontro Nacional de } \\
\text { Engenharia de } \\
\text { Sedimentos/UFSM }\end{array}$ & Grey literature \\
\hline 2000 & $\begin{array}{l}\text { CAVALCANTE, } \\
\text { A. A \& MORAIS, J. } \\
\text { O.. }\end{array}$ & $\begin{array}{c}\text { Aspectos da degradaçao ambiental } \\
\text { no baixo curso do rio Jaguaribe - } \\
\text { Uma abordagem } \\
\text { hidrossedimentológica }\end{array}$ & Jaguaribe & Sector 3 & $\begin{array}{c}\text { VI Encontro de } \\
\text { Pesquisadores / UECE }\end{array}$ & Grey literature \\
\hline 2000 & CAMPOS et al. & $\begin{array}{c}\text { Hydrological Transformations in } \\
\text { Jaguaribe River Basin during 20th } \\
\text { Century }\end{array}$ & Jaguaribe & Sector 3 & $\begin{array}{l}\text { Proceedings of the 20th } \\
\text { Annual American } \\
\text { Geophysical Union }\end{array}$ & $\begin{array}{l}\text { White } \\
\text { literature }\end{array}$ \\
\hline 2001 & $\begin{array}{l}\text { NASCIMENTO, M. } \\
\text { M., MORAIS, J.O \& } \\
\text { PINHEIRO, L. S. } \\
\end{array}$ & $\begin{array}{l}\text { Aporte sedimentar no estuário do } \\
\text { rio Malcozinhado-CE }\end{array}$ & $\begin{array}{l}\text { Malcozi- } \\
\text { nhado }\end{array}$ & Sector 3 & $\begin{array}{l}\text { X Encontro de Iniciação } \\
\text { Científica/VI Semana } \\
\text { Universitária/FUNECE }\end{array}$ & Grey literature \\
\hline 2001 & $\begin{array}{l}\text { PONTES, F. H. X. \& } \\
\text { MORAIS, J. O. }\end{array}$ & $\begin{array}{l}\text { Processos costeiros em Camocim- } \\
\text { CE }\end{array}$ & Coreaú & Sector 1 & $\begin{array}{c}\text { VII Encontro de } \\
\text { Pesquisadores / UECE }\end{array}$ & Grey literature \\
\hline 2002 & $\begin{array}{l}\text { LACERDA, L. D. \& } \\
\text { MARINS, R. V. }\end{array}$ & $\begin{array}{l}\text { River damming and changes in } \\
\text { mangrove distribution }\end{array}$ & Jaguaribe & Sector 3 & $\begin{array}{l}\text { ISME/GLOMIS } \\
\text { Electronic Journal }\end{array}$ & $\begin{array}{c}\text { White } \\
\text { literature }\end{array}$ \\
\hline 2002 & $\begin{array}{l}\text { PINHEIRO, L. S., } \\
\text { MEDEIROS, C. \& } \\
\text { MORAIS, J. O. }\end{array}$ & $\begin{array}{c}\text { Processos hidrodinâmicos e } \\
\text { morfodinâmicos no estuário do rio } \\
\text { Malcozinhado-Casvavel }\end{array}$ & $\begin{array}{l}\text { Malcozi- } \\
\text { nhado }\end{array}$ & Sector 3 & $\begin{array}{l}\text { Resumo/ Congresso } \\
\text { Brasileiro de Geologia } \\
\text { (CBG) }\end{array}$ & Grey literature \\
\hline 2002 & $\begin{array}{l}\text { PONTES, F. H. X. \& } \\
\text { MORAIS, J. O. }\end{array}$ & $\begin{array}{l}\text { Dinâmica de evolução no baixo } \\
\text { curso do rio Cauípe-Caucaia-CE }\end{array}$ & Cauípe & Sector 2 & $\begin{array}{l}\text { V Seminário do Mestrado } \\
\text { Acadêmico em Geografia } \\
\text { /UECE }\end{array}$ & Grey literature \\
\hline 2002 & PINHEIRO et al. & $\begin{array}{l}\text { Impactos hidrodinâmicos da } \\
\text { construção de barragens dos } \\
\text { estuários: área piloto rio } \\
\text { Malcozinhado Cascavel-Ceará }\end{array}$ & $\begin{array}{l}\text { Malcozi- } \\
\text { nhado }\end{array}$ & Sector 3 & Pesquisas FUNCAP & $\begin{array}{l}\text { White } \\
\text { literature }\end{array}$ \\
\hline 2003 & $\begin{array}{l}\text { DIAS, C. B., } \\
\text { PINHEIRO, L. S. \& } \\
\text { MORAIS, J. O. }\end{array}$ & $\begin{array}{l}\text { Fluxos interativos na formação da } \\
\text { planície estuarina do rio Timonha- } \\
\text { Ceará }\end{array}$ & $\begin{array}{l}\text { Timonha/ } \\
\text { Ubatuba }\end{array}$ & Sector 1 & $\begin{array}{c}\text { IX Encontros de } \\
\text { Pesquisafores e VIII } \\
\text { Semana Universitária / } \\
\text { UECE } \\
\end{array}$ & Grey literature \\
\hline 2003 & PINHEIRO, R. D. & $\begin{array}{c}\text { Ánalise do comportamento } \\
\text { hidrodinâmico e sedimentológico } \\
\text { do estuário do rio Pirangi-CE }\end{array}$ & Pirangi & Sector 3 & $\begin{array}{c}\text { Dissertação / Geociências } \\
\text { / UFRGS }\end{array}$ & Grey literature \\
\hline 2003 & PINHEIRO, L. S. & $\begin{array}{l}\text { Riscos e gestão ambiental no } \\
\text { estuário do rio Malcozinhado, } \\
\text { Cascavel-CE }\end{array}$ & $\begin{array}{l}\text { Malcozi- } \\
\text { nhado }\end{array}$ & Sector 3 & $\begin{array}{c}\text { Tese de Doutorado / } \\
\text { UFPE }\end{array}$ & Grey literature \\
\hline 2003 & MARINS et al. & $\begin{array}{c}\text { Efeitos da açudagem no rio } \\
\text { Jaguaribe }\end{array}$ & Jaguaribe & Sector 3 & Revista Ciência Hoje & $\begin{array}{c}\text { White } \\
\text { literature }\end{array}$ \\
\hline 2004 & $\begin{array}{l}\text { MORAIS, J. O., } \\
\text { PAULA, D. P. \& } \\
\text { PINHEIRO, L. S. }\end{array}$ & $\begin{array}{l}\text { Caracterização hidrodinâmica e } \\
\text { hidrológica do estuário do rio } \\
\text { Jaguaribe, CE }\end{array}$ & Jaguaribe & Sector 3 & Resumo/ I CBO & Grey literature \\
\hline
\end{tabular}


(continuação Tabela 1)

\begin{tabular}{|c|c|c|c|c|c|c|}
\hline 2004 & $\begin{array}{l}\text { GOMES, M. L. \& } \\
\text { MORAIS, J. O. }\end{array}$ & $\begin{array}{l}\text { Aspectos hidrodinâmicos, } \\
\text { sedimentológicos e impactos } \\
\text { ambientais na lagoa do Catú }\end{array}$ & Catú & Sector 2 & Resumo/ I CBO & Grey literature \\
\hline 2004 & $\begin{array}{l}\text { MORAIS, J. O., } \\
\text { DIAS, C. B. \& } \\
\text { PINHEIRO, L. S. }\end{array}$ & $\begin{array}{c}\text { Padrões de sedimentaçao da } \\
\text { planície estuarina do rio Timonha/ } \\
\text { Ubatuba-CE }\end{array}$ & $\begin{array}{l}\text { Timonha/ } \\
\text { Ubatuba }\end{array}$ & Sector 1 & Resumo/ I CBO & Grey literature \\
\hline 2004 & $\begin{array}{l}\text { FALCÂO, T. O., } \\
\text { PINHEIRO, L. S. \& } \\
\text { MORAIS, J. O. }\end{array}$ & $\begin{array}{l}\text { Caracterização sedimentológica e } \\
\text { transportes de sedimentos em } \\
\text { suspensão no estuário do rio Catú }\end{array}$ & Catú & Sector 2 & Resumo/ I CBO & Grey literature \\
\hline 2004 & $\begin{array}{l}\text { FALCÂO, T. O., } \\
\text { PINHEIRO, L. S. \& } \\
\text { MORAIS, J. O. }\end{array}$ & $\begin{array}{l}\text { Distribuição termohalina do } \\
\text { estuário do rio Catu, Aquiraz }\end{array}$ & Catú & Sector 2 & Resumo/ I CBO & Grey literature \\
\hline 2004 & $\begin{array}{l}\text { PINHEIRO, L. S., } \\
\text { MEDEIROS, C. \& } \\
\text { MORAIS, J. O. }\end{array}$ & $\begin{array}{c}\text { Processos hidrodinâmicos e } \\
\text { variação temporal dos campos de } \\
\text { salinidade no estuário do rio } \\
\text { Malcozinhado }\end{array}$ & $\begin{array}{l}\text { Malcozi- } \\
\text { nhado }\end{array}$ & Sector 3 & Resumo/ I CBO & Grey literature \\
\hline 2004 & $\begin{array}{l}\text { MORAIS, J. O., } \\
\text { PINHEIRO, L. S., } \\
\text { \&MEDEIROS, C. }\end{array}$ & $\begin{array}{c}\text { Aspectos hidrológicos e } \\
\text { hudrodinâmicos do sistema } \\
\text { estuarino Timonha/Ubatuba }\end{array}$ & $\begin{array}{l}\text { Timonha/ } \\
\text { Ubatuba }\end{array}$ & Sector 1 & Resumo/ I CBO & Grey literature \\
\hline 2004 & PINHEIRO et al. & $\begin{array}{l}\text { Estimativa da carga de material } \\
\text { particulado em suspensão e os } \\
\text { impactos de barramentos nos } \\
\text { processos de sedimentação no } \\
\text { estuário do rio Malcozinhado }\end{array}$ & $\begin{array}{l}\text { Malcozi- } \\
\text { nhado }\end{array}$ & Sector 3 & Resumo/ I CBO & Grey literature \\
\hline 2004 & $\begin{array}{l}\text { MORAIS, J. O., } \\
\text { PAULA, D. P. \& } \\
\text { PINHEIRO, L. S. }\end{array}$ & $\begin{array}{c}\text { Hidrologia e hidrodinâmica } \\
\text { estuarina aplicada ao diagnóstico } \\
\text { de riscos ambientais no estuário do } \\
\text { rio Jaguaribe, CE }\end{array}$ & Jaguaribe & Sector 3 & $\begin{array}{c}\text { IV Semana Universtária / } \\
\text { UECE }\end{array}$ & Grey literature \\
\hline 2004 & $\begin{array}{l}\text { CAVALCANTE, A. } \\
\text { A. \& MORAIS, J. O. }\end{array}$ & $\begin{array}{l}\text { Impactos na bacia hidrográfica do } \\
\text { rio Jaguaribe-CE }\end{array}$ & Jaguaribe & Sector 3 & Resumo/ I CBO & Grey literature \\
\hline 2005 & $\begin{array}{l}\text { PAULA, D. P., } \\
\text { MORAIS J. O. \& } \\
\text { PINHEIRO, L. S. }\end{array}$ & $\begin{array}{c}\text { Aspectos termohalinos e } \\
\text { ambientias do estuário do rio } \\
\text { Jaguaribe, CE }\end{array}$ & Jaguaribe & Sector 3 & $\begin{array}{l}\text { XI Simpósio Brasileiro de } \\
\text { Geografia Física Aplicada }\end{array}$ & Grey literature \\
\hline 2005 & $\begin{array}{l}\text { PAULA, D. P., } \\
\text { MORAIS, J. O. \& } \\
\text { PINHEIRO, L. S. }\end{array}$ & $\begin{array}{l}\text { Impactos da implantação de } \\
\text { barragens sobre o sistema } \\
\text { estuarino do rio Jaguaribe }\end{array}$ & Jaguaribe & Sector 3 & $57^{\mathrm{a}} \mathrm{SBPC}$ & Grey literature \\
\hline 2005 & DIAS, C. B & $\begin{array}{c}\text { Dinâmica do sistema estuarino } \\
\text { Timonha/Ubatuba: Considerações } \\
\text { Ambientais }\end{array}$ & $\begin{array}{l}\text { Timonha/ } \\
\text { Ubatuba }\end{array}$ & Sector 1 & $\begin{array}{c}\text { Dissertação/ Labomar/ } \\
\text { UFC }\end{array}$ & Grey literature \\
\hline 2005 & $\begin{array}{l}\text { PAULA, D. P., } \\
\text { MORAIS J. O. \& } \\
\text { PINHEIRO, L. S. }\end{array}$ & $\begin{array}{c}\text { Circulation and mixture process } \\
\text { and environmental impacts in the } \\
\text { Jaguaribe river estuary, CE }\end{array}$ & Jaguaribe & Sector 3 & Resumo/CARICOSTAS & Grey literature \\
\hline 2006 & $\begin{array}{l}\text { SUCUPIRA, P. A. } \\
\text { P., PINHEIRO, L. S. } \\
\text { \& ROSA, M. DE F. }\end{array}$ & $\begin{array}{l}\text { Caracterização morfométrica do } \\
\text { médio e baixo curso do rio Acaraú- } \\
\text { CE-Brasil }\end{array}$ & Acaraú & Sector 1 & $\begin{array}{l}\text { Resumo expandido/ } \\
\text { SINAGEO }\end{array}$ & Grey literature \\
\hline 2006 & $\begin{array}{l}\text { DIAS, C. B. , } \\
\text { MORAIS, J. O. \& } \\
\text { PINHEIRO, L. S. }\end{array}$ & $\begin{array}{l}\text { The effect of tide on the saline } \\
\text { structure in the Timonha/Ubatuba } \\
\text { estuarine system. }\end{array}$ & $\begin{array}{l}\text { Timonha/ } \\
\text { Ubatuba }\end{array}$ & Sector 1 & $\begin{array}{l}\text { Tropical Oceanography } \\
\text { (online) }\end{array}$ & $\begin{array}{l}\text { White } \\
\text { literature }\end{array}$ \\
\hline 2006 & PAULA, D. P. & $\begin{array}{c}\text { Elaboração de Paisagens contíguas } \\
\text { ao estuário do rio Jaguaribe-CE }\end{array}$ & Jaguaribe & Sector 3 & $\begin{array}{c}\text { Dissertação / Geografia / } \\
\text { UECE }\end{array}$ & Grey literature \\
\hline 2006 & $\begin{array}{l}\text { PAULA, D. P., } \\
\text { MORAIS J. O. \& } \\
\text { PINHEIRO, L. S. }\end{array}$ & $\begin{array}{c}\text { Análise geoambiental do estuário } \\
\text { do rio Jaguaribe-CE: tensores } \\
\text { naturais e antrópicos }\end{array}$ & Jaguaribe & Sector 3 & Resumo /SINAGEO & Grey literature \\
\hline 2006 & $\begin{array}{l}\text { MOLISANI, M. M., } \\
\text { CRUZ,A. L. V. \& } \\
\text { MAIA, L. P. }\end{array}$ & $\begin{array}{c}\text { Estimation of the freshwater river } \\
\text { discharge to estuaries in Ceará } \\
\text { State, Brazil }\end{array}$ & $\begin{array}{l}\text { Todos } \\
\text { estuários }\end{array}$ & $\begin{array}{c}\text { All } \\
\text { sectors }\end{array}$ & $\begin{array}{c}\text { Arquivos Ciências do } \\
\text { Mar / UFC }\end{array}$ & $\begin{array}{l}\text { White } \\
\text { literature }\end{array}$ \\
\hline 2006 & BEZERRA, R. G. & $\begin{array}{l}\text { Hidrodinâmica do estuário do rio } \\
\text { Choró (Cascavel / Beberibe) }\end{array}$ & Choró & Sector 3 & $\begin{array}{c}\text { Dissertação / Geologia / } \\
\text { UFC }\end{array}$ & Grey literature \\
\hline 2007 & $\begin{array}{l}\text { MUSSI, M. M., } \\
\text { CAVALCANTE, } \\
\text { M. D.; MAIA, L. P. }\end{array}$ & $\begin{array}{l}\text { Effects of water management on } \\
\text { hydrology and water quality if a } \\
\text { seni-arid in the northeast of Brazil }\end{array}$ & Cocó & Sector 2 & $\begin{array}{c}\text { Brazilian Journal of } \\
\text { Aquatic Science and } \\
\text { Tehnology }\end{array}$ & $\begin{array}{l}\text { White } \\
\text { literature }\end{array}$ \\
\hline 2007 & PAULA et al & $\begin{array}{l}\text { A Hidrodinâmica do estuário do } \\
\text { rio Piranji, CE-Brasil - Implicações } \\
\text { para o manejo e uso sustentável }\end{array}$ & Pirangi & Sector 3 & $\begin{array}{c}\text { Resumo expandido/ } \\
\text { ABEQUA }\end{array}$ & Grey literature \\
\hline 2007 & $\begin{array}{l}\text { PAULA, D. P., } \\
\text { MORAIS, J. O. \& } \\
\text { PINHEIRO, L. S. }\end{array}$ & $\begin{array}{l}\text { Classifiacação de estuários } \\
\text { tropicais bseada na estrtura halina } \\
\text { - o caso dos rios Pirangi e } \\
\text { Jaguaribe. }\end{array}$ & $\begin{array}{l}\text { Piranji e } \\
\text { Jaguaribe }\end{array}$ & Sector 3 & $\begin{array}{l}\text { Resumo expandido/ } \\
\text { SEMGIZC /UECE }\end{array}$ & Grey literature \\
\hline
\end{tabular}


(continuação Tabela 1)

\begin{tabular}{|c|c|c|c|c|c|c|}
\hline 2007 & $\begin{array}{l}\text { PAULA, D. P., } \\
\text { MORAIS, J. O. \& } \\
\text { PINHEIRO, L. S. }\end{array}$ & $\begin{array}{c}\text { Efeitos da sazonalidade climática } \\
\text { sobre o balanço hídrico do estuário } \\
\text { do rio Jaguaribe-CE }\end{array}$ & Jaguaribe & Sector 3 & $\begin{array}{l}\text { Resumo expandido/ } \\
\text { COLACMAR }\end{array}$ & Grey literature \\
\hline 2007 & MORAIS et al. & $\begin{array}{c}\text { Efeito da maré sobre a esteutura } \\
\text { salina do estuário do rio Pirangi, } \\
\text { Beberibe, Brasil }\end{array}$ & Pirangi & Sector 3 & $\begin{array}{l}\text { Resumo expandido/ } \\
\text { COLACMAR }\end{array}$ & Grey literature \\
\hline 2007 & $\begin{array}{l}\text { DIAS, C. B. \& } \\
\text { PAULA, D. P. }\end{array}$ & $\begin{array}{l}\text { Dinâmica de sedimentos em } \\
\text { sistemas estuarinos tropicais - o } \\
\text { caso do estuário do rio Pirangi }\end{array}$ & Pirangi & Sector 3 & $\begin{array}{l}\text { Resumo expandido/ } \\
\text { COLACMAR }\end{array}$ & Grey literature \\
\hline 2007 & TRUCCOLO et al. & $\begin{array}{l}\text { Aspectos hidrodinâmicos do } \\
\text { estuário do rio Jaguaribe (CE) } \\
\text { durante a estação seca. }\end{array}$ & Jaguaribe & Sector 3 & $\begin{array}{l}\text { Resumo expandido/ XII } \\
\text { Simpósio de Recursos } \\
\text { Hidricos do Nordeste - } \\
\text { ABRH }\end{array}$ & Grey literature \\
\hline 2007 & DIAS, F. J. S. & $\begin{array}{c}\text { Hidrodinâmica das descargas } \\
\text { fluviais para o estuário do Rio } \\
\text { Jaguaribe (CE) }\end{array}$ & Jaguaribe & Sector 3 & $\begin{array}{c}\text { Dissertação/Labomar/ } \\
\text { UFC }\end{array}$ & Grey literature \\
\hline 2008 & AUGUSTA et al. & $\begin{array}{l}\text { A hidrodinâmica e distribuição } \\
\text { sedimentar no canal estuarino do } \\
\text { rio Jaguaribe-CE }\end{array}$ & Jaguaribe & Sector 3 & $\begin{array}{l}\text { Resumo expandido/ } \\
\text { COLACMAR }\end{array}$ & Grey literature \\
\hline 2008 & MAIA et al. & $\begin{array}{c}\text { Alteração e classificação da } \\
\text { hidrodinâmica e distribuição } \\
\text { sedimentar do canal estuarino do } \\
\text { rio Jaguaribe-CE }\end{array}$ & Jaguaribe & Sector 3 & $\begin{array}{l}\text { Resumo expandido/ } \\
\text { CBO }\end{array}$ & Grey literature \\
\hline 2008 & $\begin{array}{l}\text { PAULA, D. P., } \\
\text { MORAIS, J. O. \& } \\
\text { PINHEIRO, L. S. }\end{array}$ & $\begin{array}{c}\text { Dinâmica de estuários tropicais } \\
\text { dominados por marés - estudo de } \\
\text { caso dos estuários inseridos no } \\
\text { litoral leste do Ceará }\end{array}$ & Jaguaribe & Sector 3 & $\begin{array}{l}\text { Resumo expandido/ } \\
\text { CBO }\end{array}$ & Grey literature \\
\hline 2008 & $\begin{array}{l}\text { QUINTELA, T. O. } \\
\text { F. }\end{array}$ & $\begin{array}{c}\text { A dinâmica ambiental do estuário } \\
\text { do rio Curu-CE: subdsídos para o } \\
\text { monitoramento e gerenciamento } \\
\text { da APA }\end{array}$ & Curu & Sector 1 & $\begin{array}{c}\text { Dissertação / Geografia / } \\
\text { UECE }\end{array}$ & Grey literature \\
\hline 2009 & $\begin{array}{l}\text { MORAIS, J. O., } \\
\text { PAULA, D. P., \& } \\
\text { PINHEIRO, L. S. }\end{array}$ & $\begin{array}{c}\text { Circulation and mixture processes } \\
\text { on the Jaguaribe River estuarine } \\
\text { waters, CE,Brasil }\end{array}$ & Jaguaribe & Sector 3 & Resumo/ABEQUA & Grey literature \\
\hline 2009 & $\begin{array}{l}\text { PAULA, D. P., } \\
\text { MORAIS, J. O. \& } \\
\text { PINHEIRO, L. S. }\end{array}$ & $\begin{array}{c}\text { Impactos de eventos naturais e } \\
\text { antrópicos em estuários tropicais - } \\
\text { O estudo de caso do estuário do } \\
\text { rio Jaguaribe }\end{array}$ & Jaguaribe & Sector 3 & $\begin{array}{l}\text { Resumo expandido/ } \\
\text { ABEQUA }\end{array}$ & Grey literature \\
\hline 2009 & $\begin{array}{l}\text { CAVALCANTE, A. } \\
\text { A. \& CUNHA, S. B. }\end{array}$ & $\begin{array}{c}\text { Caracterização do sistema fluvial } \\
\text { do rio Jaguaribe no semi-árido } \\
\text { cearense }\end{array}$ & Jaguaribe & Sector 3 & $\begin{array}{c}\text { Resumo expandido/ XII } \\
\text { Simpósio Brasileiro } \\
\text { Recursos Hidricos - } \\
\text { ABRH }\end{array}$ & Grey literature \\
\hline 2009 & $\begin{array}{l}\text { BEZERRA, M. B., } \\
\text { MORAIS, J. O. \& } \\
\text { PAULA, L. F. S. }\end{array}$ & $\begin{array}{l}\text { Barragem de Pedrinhas e sua } \\
\text { relação com a dinâmica fluvial }\end{array}$ & Jaguaribe & Sector 3 & $\begin{array}{l}\text { Resumo/ SVI Semana } \\
\text { Universitária }\end{array}$ & Grey literature \\
\hline 2009 & $\begin{array}{l}\text { DIAS, F. J. S., } \\
\text { MARINS, R. V. \& } \\
\text { MAIA, L. P. }\end{array}$ & $\begin{array}{c}\text { Hydrology of a well-mixed estuary } \\
\text { at the semi-arid Northeastern } \\
\text { Brazilian coast }\end{array}$ & Jaguaribe & Sector 3 & $\begin{array}{l}\text { Acta Limnológica } \\
\text { Brasiliensia }\end{array}$ & $\begin{array}{l}\text { White } \\
\text { literature }\end{array}$ \\
\hline 2009 & $\begin{array}{l}\text { PAULA, D. P., } \\
\text { MORAIS, J. O. \& } \\
\text { PINHEIRO, L. S. }\end{array}$ & $\begin{array}{c}\text { Longitudinal suspended sediments } \\
\text { transport in the Jaguaribe river } \\
\text { estuary }\end{array}$ & Jaguaribe & Sector 3 & $\begin{array}{c}\text { Arquivos Ciências do } \\
\text { Mar / UFC }\end{array}$ & $\begin{array}{l}\text { White } \\
\text { literature }\end{array}$ \\
\hline 2010 & $\begin{array}{l}\text { PINHEIRO, L. S, } \\
\text { MORAIS, J. O., } \\
\text { PAULA, D. P. }\end{array}$ & $\begin{array}{c}\text { Dinâmica de sedimentos em } \\
\text { suspensão em estuários sob efeito } \\
\text { da semiaridez no estado do Ceará: } \\
\text { o caso do rio Malcozinhado } \\
\end{array}$ & $\begin{array}{l}\text { Malcozi- } \\
\text { nhado }\end{array}$ & Sector 3 & $\begin{array}{c}\text { Resumo } / 45^{\circ} \text { Congresso } \\
\text { Brasileiro de Geologia }\end{array}$ & Grey literature \\
\hline 2010 & DIAS et al. & $\begin{array}{l}\text { Comparative analysis of rating } \\
\text { curve and ADP estimates of } \\
\text { instanteneous water discharge } \\
\text { through estuaries in two } \\
\text { constrating BR rivers }\end{array}$ & Jaguaribe & Sector 3 & Hydrology process & $\begin{array}{l}\text { White } \\
\text { literature }\end{array}$ \\
\hline 2010 & FREITAS et al. & $\begin{array}{c}\text { Distribuição longitudinal da } \\
\text { salinidade nos estuários dos rios } \\
\text { Jaguaribe, Pirangi e Pacoti,CE }\end{array}$ & $\begin{array}{l}\text { Pacoti, } \\
\text { Pirangi e } \\
\text { Jaguaribe }\end{array}$ & Sector 2 & $\begin{array}{l}\text { Resumo expandido/ } \\
\text { SBPC }\end{array}$ & Grey literature \\
\hline 2010 & $\begin{array}{l}\text { CARMO, F. B. M., } \\
\text { FREITAS, P. P. \& } \\
\text { SCHETTINI, C. A. } \\
\text { F. }\end{array}$ & $\begin{array}{c}\text { Distribuição vertical da salinidade } \\
\text { do estuário do rio Pacoti,CE. }\end{array}$ & Pacoti & Sector 2 & $\begin{array}{l}\text { Resumo simples/ XXIX } \\
\text { Encontro de Iniicação } \\
\text { Científica }\end{array}$ & Grey literature \\
\hline
\end{tabular}


(continuação Tabela 1)

\begin{tabular}{|c|c|c|c|c|c|c|}
\hline 2010 & FREITAS et al. & $\begin{array}{c}\text { Comportamento da maré } \\
\text { astronômica no estuário do rio } \\
\text { Pacoti,CE }\end{array}$ & Pacoti & Sector 2 & $\begin{array}{l}\text { Resumo simples /XXIII } \\
\text { SNO }\end{array}$ & Grey literature \\
\hline 2010 & $\begin{array}{l}\text { FREITAS, P. P., } \\
\text { CARMO, F. B. M \& } \\
\text { SCHETTINI, C. A. } \\
\text { F. }\end{array}$ & $\begin{array}{c}\text { Oceanografia Física do Estuário do } \\
\text { Rio Pacoti,CE }\end{array}$ & Pacoti & Sector 2 & $\begin{array}{l}\text { Resumo simples/ XXIX } \\
\text { Encontro de Iniicação } \\
\text { Científica }\end{array}$ & Grey literature \\
\hline 2010 & FROTA et al. & $\begin{array}{c}\text { Avaliação da estratificação do } \\
\text { estuário do rio Jaguaribe }\end{array}$ & Jaguaribe & Sector 3 & $\begin{array}{c}\text { Resumo expandido/ } \\
\text { SBPC }\end{array}$ & Grey literature \\
\hline 2010 & $\begin{array}{l}\text { PINHEIRO, L. S. \& } \\
\text { MORAIS, J. O. }\end{array}$ & $\begin{array}{l}\text { Interferência de barramentos no } \\
\text { regime hidrológico do estuário do } \\
\text { rio Catú-CE-Nordeste do Brasil }\end{array}$ & Catú & Sector 2 & Sociedade \& Natureza & $\begin{array}{l}\text { White } \\
\text { literature }\end{array}$ \\
\hline 2011 & $\begin{array}{l}\text { FREITAS, P. P., } \\
\text { CARMO, F. B. M \& } \\
\text { SCHETTINI, C. A. } \\
\text { F. }\end{array}$ & $\begin{array}{c}\text { Comportamento da maré e da } \\
\text { salinidade longitudinal no estuário } \\
\text { do rio Pacoti }\end{array}$ & Pacoti & Sector 2 & $\begin{array}{l}\text { Resumo simples / XXIII } \\
\text { SNO }\end{array}$ & Grey literature \\
\hline 2011 & $\begin{array}{l}\text { FALCÃO, T. O., } \\
\text { MORAIS, J. O. \& } \\
\text { PINHEIRO, L. S. }\end{array}$ & $\begin{array}{c}\text { Morphodynamics of the Curu } \\
\text { Estuary Inlet-Brazil }\end{array}$ & Curu & Sector 1 & $\begin{array}{l}\text { Journal of Coastal } \\
\text { Research (JCR) }\end{array}$ & $\begin{array}{l}\text { White } \\
\text { literature }\end{array}$ \\
\hline 2011 & BEZERRA et al. & $\begin{array}{c}\text { Levantamento hidrográfico da } \\
\text { região estuarina do rio Jaguaribe } \\
\text { (NE-Brasil) entre os anos de } 2004 \text { e } \\
2009 .\end{array}$ & Jaguaribe & Sector 3 & $\begin{array}{l}\text { V Simpósio Brasileiro de } \\
\text { Oceanografia }\end{array}$ & Grey literature \\
\hline 2011 & CARMO et al. & $\begin{array}{c}\text { Distribuição vertical da salinidade } \\
\text { nos estuários dos rios Pacoti e } \\
\text { Pirangi, CE }\end{array}$ & $\begin{array}{l}\text { Pacoti e } \\
\text { Pirangi }\end{array}$ & $\begin{array}{l}\text { Sector } 2 \\
\text { and } \\
\text { Sector } 3\end{array}$ & $\begin{array}{l}\text { Resumo simples /XXIII } \\
\text { SNO }\end{array}$ & Grey literature \\
\hline 2011 & $\begin{array}{l}\text { RODRIGUES, S. W. } \\
\text { P. \& SOUZA } \\
\text { FILHO, P. W. M. }\end{array}$ & $\begin{array}{c}\text { Detecção da emudança no } \\
\text { manguezal ao longo do rio } \\
\text { Coreaú-CE através da análise } \\
\text { multitemporal em imagens TM/ } \\
\text { LANDSAT-5 no periódo de } 1985 \text { a } \\
2008 \text {. }\end{array}$ & Coreaú & Sector 1 & $\begin{array}{l}\text { Resumo expandido/ } \\
\text { ABEQUA }\end{array}$ & Grey literature \\
\hline 2011 & DIAS et al. & $\begin{array}{l}\text { Caracteristicas hidrodinâmicas da } \\
\text { circulação estuarina do rio } \\
\text { Jaguaribe nas interfaces ZR/ZM e } \\
\text { ZM/ZC durante a estação chuvosa } \\
\text { de } 2009 \text {. }\end{array}$ & Jaguaribe & Sector 3 & COLACMAR & Grey literature \\
\hline 2011 & $\begin{array}{l}\text { DIAS, F. J. S., } \\
\text { CASTRO, B. M. \& } \\
\text { LACERDA, L. D. }\end{array}$ & $\begin{array}{c}\text { Balanço líquido e taxa de descarga } \\
\text { (flushing time) do estuário do rio } \\
\text { Jaguaribe durante um período de } \\
\text { grandes descargas fluviais }\end{array}$ & Jaguaribe & Sector 3 & COLACMAR & Grey literature \\
\hline 2011 & DIAS et al. & $\begin{array}{l}\text { Balanço de massa do material em } \\
\text { suspensão na interface continente- } \\
\text { oceano do estuário do rio } \\
\text { Jaguaribe (CE) durante um } \\
\text { período de grande descarga fluvial }\end{array}$ & Jaguaribe & Sector 3 & $\begin{array}{c}\text { XIII Congresso Brasileiro } \\
\text { de Geoquimica }\end{array}$ & Grey literature \\
\hline 2011 & $\begin{array}{l}\text { PINHEIRO, L. S. \& } \\
\text { MORAIS, J. O. }\end{array}$ & $\begin{array}{c}\text { The effect of semi-aridity and } \\
\text { damming on sedimentary } \\
\text { dynamics in estuaries- } \\
\text { Northeastern region of Brazil }\end{array}$ & $\begin{array}{l}\text { Jaguaribe, } \\
\text { Timonha, } \\
\text { Acaraú e } \\
\text { Malcozi- } \\
\text { nhado }\end{array}$ & $\begin{array}{c}\text { All } \\
\text { sector }\end{array}$ & $\begin{array}{l}\text { Journal of Coastal } \\
\text { Research (JCR) }\end{array}$ & $\begin{array}{l}\text { White } \\
\text { literature }\end{array}$ \\
\hline 2012 & $\begin{array}{l}\text { COSTA, C. A. \& } \\
\text { PINHEIRO, L. S. }\end{array}$ & $\begin{array}{c}\text { Dinâmica fluvial de rios } \\
\text { semiáridos: um estudo de caso da } \\
\text { bacia do médio/baixo Jaguaribe- } \\
\text { CE }\end{array}$ & Jaguaribe & Sector 3 & Resumo / IX SINAGEO & Grey literature \\
\hline 2012 & $\begin{array}{l}\text { SILVA, D. B. M., } \\
\text { CAMELO, C. E. C. } \\
\text { J. \& PINHEIRO, L. } \\
\text { S. }\end{array}$ & $\begin{array}{l}\text { Morfodinâmica da desembocadura } \\
\text { do estuário do rio Pacoti- } \\
\text { CE:Evolução e impactos } \\
\text { ambientais em regimes semi- } \\
\text { áridos sob efeito de açudagem }\end{array}$ & Pacoti & Sector 2 & $\begin{array}{l}\text { XXXI Encontro de } \\
\text { Iniciação Científica }\end{array}$ & Grey literature \\
\hline 2012 & $\begin{array}{l}\text { SILVA, A. C., } \\
\text { LACERDA, L. D. \& } \\
\text { MORAIS, J. O. }\end{array}$ & $\begin{array}{c}\text { Spatial and Temporal Variability } \\
\text { of Hydrographic parameters } \\
\text { measured in the estuary of the } \\
\text { Jaguaribe River }\end{array}$ & Jaguaribe & Sector 3 & $\begin{array}{l}\text { Revista Brasileira de } \\
\text { Geofísica (RBGf) }\end{array}$ & $\begin{array}{c}\text { White } \\
\text { literature }\end{array}$ \\
\hline 2012 & FREITAS, P. P. & $\begin{array}{l}\text { Aspectos hidrodinâmicos do } \\
\text { estuário do rio Cocó }\end{array}$ & Cocó & Sector 2 & $\begin{array}{c}\text { Trabalho de Conclusão } \\
\text { de Curso }\end{array}$ & Grey literature \\
\hline
\end{tabular}


(continuação Tabela 1)

\begin{tabular}{|c|c|c|c|c|c|c|}
\hline 2012 & $\begin{array}{l}\text { VALENTIM, S. S., } \\
\text { BEZERRA, M. O. } \\
\text { M. \& MORAIS, J. } \\
\text { O. }\end{array}$ & $\begin{array}{l}\text { Dinâmica de um estuário de } \\
\text { mesomaré na costa semiárida } \\
\text { equatorial (Ceará, Brasil) }\end{array}$ & Coreaú & Sector 1 & Resumo/CBO & Grey literature \\
\hline 2013 & $\begin{array}{l}\text { CAMELO, C. E. C. } \\
\text { J., ROCHA, M. N. } \\
\text { \& PINHEIRO, L. S., }\end{array}$ & $\begin{array}{c}\text { Influência do transporte eólico na } \\
\text { evolução da desembocacura do } \\
\text { estuário do rio Coreaú, CE }\end{array}$ & Coreaú & Sector 1 & $\begin{array}{l}\text { Resumo/ Encontros } \\
\text { Universitários UFC }\end{array}$ & Grey literature \\
\hline 2013 & $\begin{array}{l}\text { SILVA, D. B. M., } \\
\text { PINHEIRO, L. S., } \\
\text { MORAIS, J. O.. }\end{array}$ & $\begin{array}{c}\text { Caracterização Morfológica do } \\
\text { estuário do rio Coreaú }\end{array}$ & Coreaú & Sector 1 & Resumo/ABEQUA & Grey literature \\
\hline 2013 & ROCHA, G. H. M. & $\begin{array}{c}\text { Morfodinâmica de } \\
\text { desembocaduras de sistemas } \\
\text { estuarinos barrados: o caso do rio } \\
\text { Pacotei, CE }\end{array}$ & Pacoti & Sector 2 & $\begin{array}{c}\text { Dissertação / Geografia / } \\
\text { UECE }\end{array}$ & Grey literature \\
\hline 2013 & $\begin{array}{c}\text { CAMELO, C. E. C. } \\
\text { J. }\end{array}$ & $\begin{array}{c}\text { Avaliação da influência do } \\
\text { transporte eólico na evolição da } \\
\text { desembocacura do estuário do rio } \\
\text { Coreaú }\end{array}$ & Coreaú & Sector 1 & TCC & Grey literature \\
\hline 2014 & SILVA, D. B. M. & $\begin{array}{c}\text { Estudo da Morfodinâmica e } \\
\text { morfologia de fundo da } \\
\text { desembocadura do estuário do rio } \\
\text { Coreaú }\end{array}$ & Coreaú & Sector 1 & $\mathrm{TCC}$ & Grey literature \\
\hline 2014 & $\begin{array}{l}\text { VALENTIM, S. S., } \\
\text { BEZERRA, M. O. } \\
\text { M. \& VALLE- } \\
\text { LEVINSON, A. }\end{array}$ & $\begin{array}{l}\text { Hydrography in a hypersaline } \\
\text { tropical estuary }\end{array}$ & Coreaú & Sector 1 & $\begin{array}{c}\text { Resumo expandido/ } \\
\text { PECS }\end{array}$ & Grey literature \\
\hline 2014 & $\begin{array}{l}\text { MORAIS, J. O., } \\
\text { DIAS, C. B. \& } \\
\text { PINHEIRO, L. S. }\end{array}$ & $\begin{array}{l}\text { Capacidade de depuração do } \\
\text { sistema estuarino Timonha- } \\
\text { Ubatuba, no Estado do Ceará, } \\
\text { Brasil }\end{array}$ & $\begin{array}{l}\text { Timonha/ } \\
\text { Ubatuba }\end{array}$ & Sector 1 & $\begin{array}{c}\text { Arquivos Ciências do } \\
\text { Mar / UFC }\end{array}$ & $\begin{array}{l}\text { White } \\
\text { literature }\end{array}$ \\
\hline 2014 & $\begin{array}{l}\text { SCHETTINI, C. A. } \\
\text { F., BEZERRA, L. J. } \\
\text { C. \& TRUCCOLO, } \\
\text { E. C. }\end{array}$ & $\begin{array}{l}\text { Distribuição longitudinal da } \\
\text { salinidade em estuários de baixo } \\
\text { influxo no Ceará. }\end{array}$ & $\begin{array}{l}\text { Pacoti, } \\
\text { Pirangi e } \\
\text { Jaguaribe }\end{array}$ & $\begin{array}{l}\text { Sector } 2 \\
\text { and } \\
\text { Sector } 3\end{array}$ & $\begin{array}{l}\text { Resumo expandido/ XII } \\
\text { Simpósio de Recursos } \\
\text { Hidricos do Nordeste - } \\
\text { ABRH } \\
\end{array}$ & Grey literature \\
\hline 2014 & COLARES et al. & $\begin{array}{c}\text { Distribuição sedimentar e } \\
\text { hidrodinâmica de um estuário } \\
\text { tropical }\end{array}$ & Coreaú & Sector 1 & $\begin{array}{c}\text { Arquivos Ciências do } \\
\text { Mar / UFC }\end{array}$ & $\begin{array}{l}\text { White } \\
\text { literature }\end{array}$ \\
\hline 2015 & COLARES, M. C. S. & $\begin{array}{c}\text { Evolução geomorfológica do canal } \\
\text { estuarino do rio Coreaú, Camocim, } \\
\text { Ceará, Brasil }\end{array}$ & Coreaú & Sector 1 & $\begin{array}{l}\text { Tese de Doutorado / } \\
\text { LABOMAR/UFC }\end{array}$ & Grey literature \\
\hline
\end{tabular}

\section{Type of Literature and repercussion}

Undergraduate courses were institutionalized in Brazil since 1970s by 5.540/68 law. After that, bachelor's courses became the main players of Brazilian scientific production. On academic community, whereas career progress at universities and institutes considers scientific production index as evaluation, students are pushed to publish since early terms. The knowledge produced at university is usually launched on some communication channels that differ a study area to another, or from research to another. Formal publications (e.g. abstracts, reports, and thesis) are considered driving force to academic community since publications seeks to developing the system, promoting the knowledge generation cycle (Cavalcanti, 2000). However, these formal publications are generally written in local languages and limit the international repercussion of the research.

This paper is focused on classification per type of literature: Grey literature and White literature. Grey literature is defined as documents produced on all levels of government, academics 
(symposiums, conferences, meetings, and thesis), business and industry in print and electronic formats, but not managed by commercial publishers (Alberani, 1990; GL'99 Conference Program, 1999). Usually, grey literature have difficult access (technical reports, abstract or texts published in proceedings), or published at one specific language (other than English) which limits its wider publicity and knowledge. White Literature is considered publications in articles in periodicals with high repercussion on academic community.

\section{METHODS}

This paper to choose investigate which scientists or research groups that more published about estuaries in the Ceará state, with focus in physical oceanography area. Many keywords as

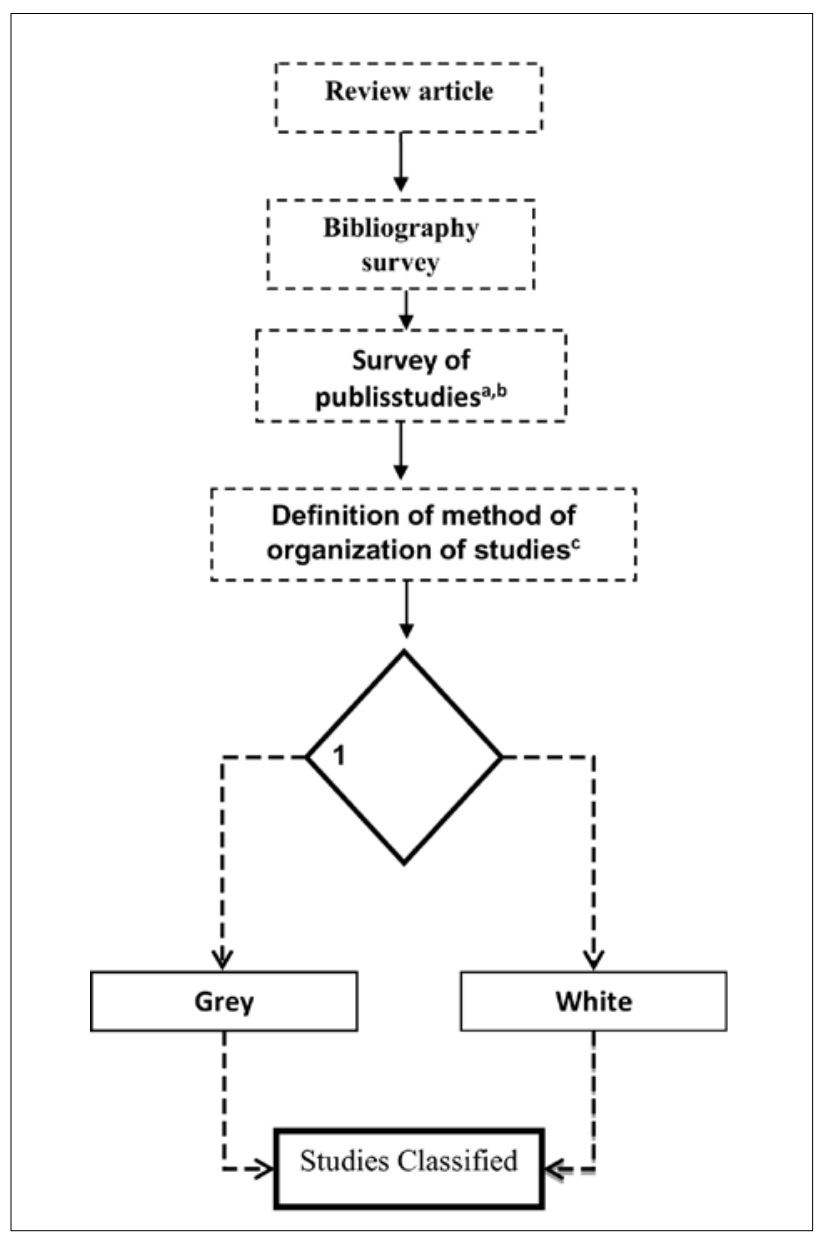

Figure 1 - Classification flowchart of studies by type of literature.a Studies carried out in the estuaries of Ceará state with focus on Physical Oceanography; $b$ Total of 100 publications from the period 1974-2015; c Type of document, Location of the estuary, Classification by type of literature. 1 - Grey (Monograph, Master Thesis, PhD Thesis, Academic events, e.g. abstract); 2 - White (Papers). names of the estuaries and main terms of area (e.g. "Hydrodynamic", "Hydrology", "Dam built", "Salinity profile", "Current velocity") have been used to facilitate the selection of studies as well as any websites (e.g. capes periodics, science direct). Resulting in total of 100 publications available online: academic events (abstracts, extended abstracts, dissertations (master's thesis), thesis (PhD) and articles) (Figure 1). Each study was divided by year, author, title, name of estuary, location based on sector of Ceará State, publication media, and type of literature.

Further, it was researched the classification by WebQualis/CAPES referring to each periodic that studies were published.

\section{RESULTS}

The main product of this research was to quantify a maximum number of studies and to classify by location and type of literature (Table 1). This research was developed to facilitate future studies, with a compilation of existing studies to serve as background. Table 1 exhibits all publications selected. The distribution of the studies are in chronological order and dates from 1974 to 2015. The elaboration of inventory identified one main research group that publish and develop studies about estuaries in Ceará State - Laboratory of Geology and Geomorphology coastal and oceanic at State University of Ceará (LGCO/UECE) - that have principal focus with geomorphology, sediment transports, and hydrology aspects.

Among the selected publications of publications selected, $77 \%$ are considered Grey literature and 23\% White literature (Table 2). Jaguaribe River is main hydric system of Ceará state and according to inventory (Table 1 ) were 42 studies performed on it.

According to Figure 2 only eleven studies were published before 2000 and eighty nine studies after 2000, from which it can be inferred that regional scientific production has been extensively developed until recently. These data are the result of more investment in education in the last decades, with greater availability of instruments for field works, research projects and research groups. However, can be identify number decreasing of publications in last year that refers to a possible change of focus of studies of the research groups and studies in the phase of elaboration.

The table 2 shows distribution of type of document (publications) over sector coastal of Ceará 
Table 2 - Distribution of studies per sector coastal of Ceará state.

\begin{tabular}{|l|c|c|c|c|c|c|}
\hline $\begin{array}{l}\text { Type of } \\
\text { document }\end{array}$ & $\begin{array}{c}\text { Sector 1 } \\
\text { (West } \\
\text { extreme } \\
\text { and west } \\
\text { coast) }\end{array}$ & $\begin{array}{c}\text { Sector 2 } \\
\text { (Metropolitan } \\
\text { sector of } \\
\text { Fortaleza) }\end{array}$ & $\begin{array}{c}\text { Sector 3 } \\
\text { (East } \\
\text { coast) }\end{array}$ & Others & Total & (\%) \\
\hline Paper & 5 & 7 & 9 & 2 & 23 & $23 \%$ \\
\hline Monograph & 2 & 1 & $*$ & $*$ & 3 & $3 \%$ \\
\hline $\begin{array}{l}\text { Master } \\
\text { Thesis }\end{array}$ & 3 & 1 & 4 & $*$ & 8 & $8 \%$ \\
\hline PhD Thesis & 1 & 1 & 1 & $*$ & 3 & $3 \%$ \\
\hline $\begin{array}{l}\text { Academic } \\
\text { events }\end{array}$ & 11 & 11 & 38 & 3 & 63 & $63 \%$ \\
\hline Total & 22 & 21 & 52 & 5 & 100 & $100 \%$ \\
\hline (\%) & $22 \%$ & $21 \%$ & $52 \%$ & $5 \%$ & $100 \%$ & \\
\hline
\end{tabular}

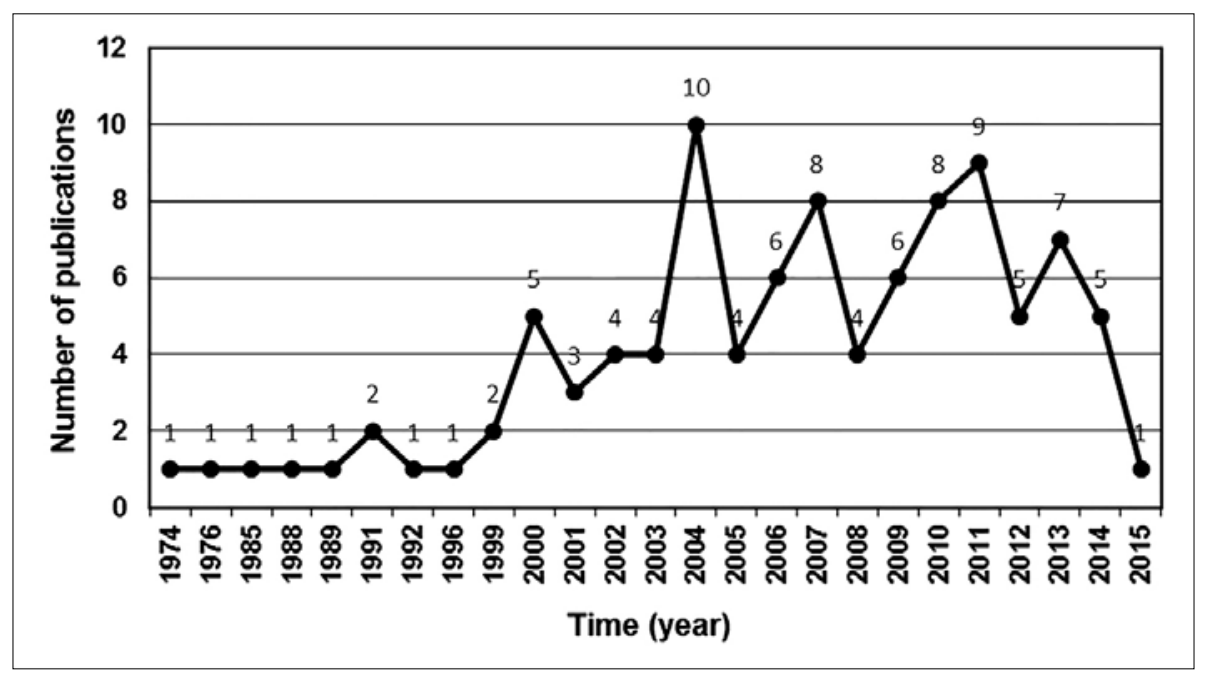

Figure 2 - Distribution of publications over time.

state. Among the main results, sectors 1 and 2 have almost the same number of studies, 22 and 21 studies, respectively. It was observed more white literature (papers) published (7) in sector 2 than in sector 1. Most studies (52) were realized in sector 3 (Sector 3), with predominance of studies published in academic events, e.g. abstract (grey literature). Also, sector 3 has more articles published in comparison to sector 1 and sector 2. However, considering all sectors of coast Ceará state, most studies have been published in academic events (63\%).

The table 3 shows the classification of each periodic based on WebQualis defined by CAPES and impact factor by its own magazine/journal. According to Table 3, most publications were published in Arquivos Ciências do Mar (6 papers or $26 \%$ of total) which owns classification of "B5", followed by Revista de Geologia (3) and Journal Coastal Research (JCR) (2).
WebQualis is the set of procedures used by CAPES to measure the quality of the intellectual production of graduate programs. It is created to evaluate the national scientific system and is based on information provided by data collection application. As a result, WebQualis provides a list of the classification of journals used by graduate programs for the dissemination of its production. Measure of quality of this production is carried out indirectly.

Thus, WebQualis assesses the quality of articles and others types of production, from the analysis of the quality of the publications media, i.e. scientific journals. The WebQualis is divided into eight (8) types, in descending order of value: A1, A2, B1, B2, B3, B4, B5 and C describe below:

- A1 - with the highest impact factor equal to or greater than 3,800 
- $\quad$ A2 - Impact Factor between 3,799 and 2,500

- $\quad$ B1 - Impact Factor between 2,499 and 1,300

- B2 - Impact Factor of 1.299 and 0.001

- B3, B4 and B5 - are indexed in MEDLINE, SCIELO, LILACS but without impact factor

- C - irrelevant, with zero weight.

According to classification of WebQualis system, periodic Hydrology process (2,696 impact factor - even A2) and Sociedade \& Natureza (B1) have better impact on scientific community and publications with classification B3 to B5 have low or no impact.

Table 3 - Classification each periodic based with WebQualis/CAPES/impact factor.

\begin{tabular}{|l|c|c|c|}
\hline Periodic & $\begin{array}{c}\text { WebQualis } \\
\text { CAPES }\end{array}$ & $\begin{array}{c}\text { Impact } \\
\text { Factor }\end{array}$ & Distribution \\
\hline Arquivos Ciências do Mar & B5 & & 6 \\
\hline Boletim de Estudos de Ciências do Mar & B5 & & 1 \\
\hline Revista Brasileira de Geofísica (impresso) & B2 & & 1 \\
\hline Journal Coastal Research & & 0.755 (2013) & 2 \\
\hline Sociedade E Natureza (UFU online) & B1 & & 1 \\
\hline Hydrology Days Publications & Nacional/A & & 1 \\
\hline Tropical Oceanography (Online) & B4 & & 1 \\
\hline Pesquisas FUNCAP & unavailable & unavailable & 1 \\
\hline Acta Limnológica Brasiliensia & B2 & & 1 \\
\hline Brazilian Journal of Aquatic Science and & B3 & & 1 \\
\hline Technology (impresso) & & & 3 \\
\hline Revista de Geologia (Fortaleza) & B4 & & 1 \\
\hline ISME/GLOMIS Electronic Journal & B4 & & 1 \\
\hline Ciência Hoje & B5 & & 1 \\
\hline Hydrological processes & & 2.696 (2013) & \\
\hline Brazilian Journal of Oceanography (impresso) & B2 & & 1 \\
\hline
\end{tabular}

\section{CONCLUSIONS}

Perform a literature review requires caution and the need to define a coherent method for classifying. This research select published studies that have focus in estuarine hydrodynamic. The conclusions are based in these outcomes presented below:

1) 100 publications selected: $77 \%$ are considered Grey Literature with low repercussion and 23\% White Literature with greater repercussion in the scientific community. The main characteristic of White Literature are the use of the English language in the articles, providing greater reach, that is, it allows a greater number of researchers to access the content of the study.

2) Among the 100 studies, only 11 were published before 2000's and 89 studies after 2000's. There was considerable increase in the number of studies after 2000's, which promoted the development of state of knowledge of estuaries. $63 \%$ was published in academic events. The last year (2015) presented a decline in published studies and the possible reason is the delay in the preparation and publication of the studies. Also, the trend is to reduce the number of studies due cuts in Education funding.

3) According to the inventory, in the total of 100 studies, most of them were developed in estuaries located in the eastern sector of the state. Of the fifty studies performed on Sector 3, forty two were conducted in Jaguaribe River. The evolution of studies in the Jaguaribe River basin is an expected fact due to the economic and environmental importance of the system, with multidisciplinary groups conducting several studies in the region. The dam built, the understanding of physical processes in estuarine dynamic, and the wealth of local ecosystems become relevant the development of research in this system. Sector 3 (East Coast) have the higher number of articles published (9) too.

4) Sector 1 (extreme west and west coast) and Sector 2 (metropolitan region of Fortaleza) have similar number of studies. Its incomprehensible the lower number of studies perfomed in estuarine systems located in the western sector of the Ceará state, however, its assumed that the logistics and costs to realized field works justify this number.

5) The main studies were published in Arquivos de Ciências do Mar (6). One of the reasons for the major number of papers published in the regional journal may be to the consolidate the quality of the journal's publications.

Its essential to highlight the evolution of the number of researches carried out in the estuaries 
of Ceará over the last decades and the several journals that studies were published (Table 3) indicate a better extension of regional researches in scientific community.

\section{REFERENCES}

Alberani, V.; Pietrangeli, P.D.C.; Mazza, A.M.R. The use of grey literature in health sciences: a preliminary survey. Bulletin of the Medical Library Association 78(4): 358-363, 1990.

Cameron, W. M. and Pritchard, D. W. Estuaries. In M. N. Hill (editor): The Sea vol. 2, p.306-324, John Wiley and Sons, New York, 1963.

Campos, J.N.B., Studart, T.M.C., Luna, R. and Franco, S.Hydrological Transformations in Jaguaribe River Basin during 20th Century. Proceedings of the 20th Annual American Geophysical Union. Fort Collins: Hydrology Days Publications, p.221-227, 2000.

Cavalcanti, I. Gonçalves Milet et al. Análise comparativa da produção científica entre as áreas sociais e tecnológicas. In: Seminário Nacional de Bibliotecas Universitárias, 10., 2000, Florianópolis, SC. Florianópolis, 2000.

Day, J. H. What is an estuary? South Africa J.ournal Science. pp.76: 198, 1980.

Dyer, K.R. Circulation and Mixing in Stratified Estuaries. Marine Chemistry, vol. 32, p.111-120. Disponible: http://dx.doi.org/10.1016/0304-4203 (91)90031-Q. 1991

Dyer, K.R. Estuaries: a physical introduction. 2nd ed. Wiley. J. 165 p. New York, 1997.

Fairbridge, R. W.The estuary: its definition and geodynamic cycle, p.1-35, in Chemistry and Biogeochemistry of Estuaries (eds. E. Olausson \& I. Cato). Wiley, New York, 1980.

GL'99 Conference Program. Fourth International Conference on Grey Literature: New Frontiers in Grey Literature.GreyNet, Grey Literature Network Service. Washington D.C. USA,1999.

Kjerfve, B.J. Comparative oceanography of coastal lagoons, p. 63-81, in Wolfe, D. A., Ed. Estuarine Variability. Academic Press, Inc., New York, 1986.
Morais, J. O.; Pinheiro, L. S. The effect of semi-aridity and damming on sedimentary dynamics in estuaries - Northeastern region of Brazil. Journal of Coastal Research, v. 64, p. 1540-1544, 2011.

Noronha, D. P.; Población, D. A. Produção das literaturas branca e cinzenta pelos docentes/ doutores dos programas de pós-gradução em ciência da informação no Brasil.. Ciência da Informação, Brasília, DF, v. 31, n.2, p. 1-19, 2002.

Pinto, A. C., Andrade, J. B. Fator de impacto de revistas científicas: Qual o significado deste parâmetro?. Química Nova, vol. 22, n 3, p.448453, 1999.

Población, D. A.; Noronha, D. P. Literatura cinzenta versus literatura branca: transição dos autores das comunicações dos eventos para produtores de artigos. Ciência da Informação, Brasília, v. 25, n.2, p. 228-242, 1996.

Población, D. A.; Noronha, D. P. Producción académica de docentes/doctores de los programas de posgrado en Ciencia de la Información en Brasil. . Ciencia de La Información, La Habana, Cuba, v. 33, n. 1, p. 25-33, 2002.

Potter IC, Chuwen BM, Hoeksema SD, Elliott M. The concept of an estuary: A definition that incorporates systems which can become closed to the ocean and hypersaline. Estuary Coast Shelf Sci 87: 497-500, 2010.

Pritchard, D. W. Estuarine circulation patterns. Proceedings of the American Society of Civil Engineers 81, no 717, 1 - 11, 1955.

Pritchard, D. W. Estuarine hydrography. Advances in Geophysics 1, 243 - 280, 1952.

Pritchard, D. W. Salt balance and exchange rate for Chincoteague Bay. Chesapeake Sci. 1:48-57, 1960.

Pritchard, D. W. The dynamics structure of a coastal plain estuary. J. Mar. Res. 15, 33-42, 1956.

Pritchard, D. W. What is an estuary, physical view point, p. 3-5, in Lauf, G. H. (editor). Estuaries. American Association for the Advancement of Science, vol. 83, Washington D.C, 1967.

Spinak, E. Indicadores cienciometricos, Brasília, Ciência da Informação, v. 27, n.2, p. 141-148, 1998. 